\title{
Editorial to computational techniques in management science
}

\author{
Panos Parpas · Wolfram Wiesemann
}

Published online: 12 November 2013

(C) Springer-Verlag Berlin Heidelberg 2013

This double issue is based on presentations at the ninth International Conference on Computational Management Science held at Imperial College London, United Kingdom. The topics covered highlight the role played by computational techniques to solve relevant real-world problems in management science.

The CMS conference is an annual meeting associated with the Journal of Computational Management Science. The aim of this conference is to provide a forum for theoreticians and practitioners from academia and industry to exchange knowledge, ideas and results in a broad range of topics relevant to the theory and practice of computational methods, models and empirical analysis for decision making in economics, engineering, finance and management. The focus is on all computational aspects of management science: theoretical and empirical studies of computational methods, models and empirical analysis. These include computational economics, finance and statistics, energy, scheduling, supply chains, design, analysis and applications of optimisation algorithms, deterministic, dynamic, stochastic, robust and combinatorial optimisation models, solution algorithms, learning and forecasting such as neural networks and genetic algorithms, models and tools of knowledge acquisition, such as data mining, and all other topics in management science with the emphasis on computational paradigms.

In the first paper, Rasmussen et al. use a stochastic programming approach to study the problem of mortgage choice and refinancing. The model is calibrated to

\footnotetext{
P. Parpas

Department of Computing, Imperial College London,

180 Queen's Gate, London SW7 2AZ, UK

W. Wiesemann $(\varangle)$

Imperial College Business School, South Kensington Campus,

London SW7 2AZ, UK

e-mail:ww@imperial.ac.uk
} 
the Danish market. Based on their empirical model, the authors find that the optimal choice is to use more than one loan. In the second paper, Cano et al. develop a model to support the decision making process of public building operators. The authors introduce a two-stage stochastic programming problem and illustrate the trade-offs between long-term and short-term decisions. In the third paper, Vizgunov et al. define a market graph for the Russian stock market and find that, contrary to the US stock markets, the most attractive Russian stocks (in terms of trading volume) have the strongest correlation between their returns. In the fourth paper, Chin and Siddiqui investigate how risk-averse power companies can optimally expand their generation capacities under demand uncertainty. To this end, they assume that the companies can hedge their risks by simultaneously acting on the spot and forward markets. The authors study the problem in a monopolistic and duopolistic setting, and they find that forward markets allow to hedge risks and improve market efficiency. In the fifth paper, Banez-Chicharro et al. study smart charging profiles for electric vehicles. The paper, which has won the CMS 2012 Best Student Paper Prize, compares different strategies for charging electric vehicles, as well as their impact on variable charging costs, emissions and the possibility to integrate renewable energy sources in the power system. The numerical results demonstrate the benefits of smart charging profiles over naive unregulated profiles. In the sixth paper, D'Amato et al. introduce a methodology to measure longevity risk that avoids structural incompleteness of existing techniques. Unlike previous methods, the authors take into account the dependence across age and time and analyze a bootstrap methodology that captures the spatial dependence in prediction intervals for mortality projection rates.

The last three papers were originally submitted for the Computational Management Science Conference in 2011. Due to space limitations in the 2011 special issue it was not possible to publish the papers there. In the seventh paper, Marshal and Shaw develop an algorithm for learning the structure of a particular class of Bayesian networks. Their algorithm is based on a greedy-search technique, and the authors evaluate their approach on a large simulated data-set. In the eighth paper, Djamal and Fatma study multiple-objective linear stochastic programs with integer decisions. The authors propose a new pivoting technique that is based on the L-shaped method and the combined method developed by Chaabane and Pirlot. The technique is applied to a two-stage stochastic integer linear program with three objectives. In the ninth paper, Kaut et al. develop a formulation for stochastic programming problems with more than one time scale. They show that the new approach drastically reduces the model size compared to the traditional formulation and present two real-life applications from energy planning.

The submissions clearly demonstrate the increasing importance of stochastic decision-making in the energy and financial markets. Due to the high complexity surrounding quantitative models, research on computational management science is likely to be of increasing significance in the future. 